\title{
Primary pulmonary leiomyosarcoma: A case report
}

\author{
XIAONA XIE ${ }^{1}$, YANFAN CHEN $^{2}$, CHENG DING $^{2}$, XIAOMING YU $^{2}$, LIZHEN ZOU $^{2}$, \\ BOTAO XU ${ }^{3}$, LIANGXING WANG ${ }^{2}$ and XIAOYING HUANG ${ }^{2}$

\begin{abstract}
${ }^{1}$ Pulmonary Division, Zhejiang University of Traditional Chinese Medicine Affiliated with Wenzhou Hospital, Wenzhou, Zhejiang 325000; ${ }^{2}$ Pulmonary Division and ${ }^{3}$ Division of General Surgery,
\end{abstract} \\ The First Affiliated Hospital of Wenzhou Medical University, Wenzhou, Zhejiang 325000, P.R. China
}

Received December 2, 2014; Accepted November 30, 2015

DOI: $10.3892 / \mathrm{ol} .2016 .4131$

\begin{abstract}
Primary pulmonary leiomyosarcoma (PPL) is an extremely rare malignant tumor. It has been revealed that PPL may originate from the smooth muscle of the pulmonary parenchyma, pulmonary arteries and bronchi. Patients with PPL may be asymptomatic or present with symptoms similar to those observed in other primary lung tumors. The present study reports the case of a 48 -year-old man who presented with a lung mass and underwent a right upper-middle lobe bronchoscope tumor resection. The patient was subsequently diagnosed with PPL. Following the bronchoscopic tumor resection, chemotherapy was administered to the patient; however, the patient succumbed to the disease after the second cycle of chemotherapy.
\end{abstract}

\section{Introduction}

Leiomyosarcomas are tumors of the smooth muscle cells that may originate in any location, but most often arise in the uterus, gastrointestinal tract and soft tissue. Primary pulmonary leiomyosarcoma (PPL) is an extremely rare malignant mesenchymal tumor that appears to originate from the smooth muscle cells of the bronchial and blood vessel wall. PPL accounts for $<0.5 \%$ of all malignant pulmonary tumors (1). A previous study reported a 5-year survival rate of $60 \%$ for PPL (2). The tumors may be treated using surgical resection, which is the primary and definitive mode of treatment. Surgical strategies consist of lobectomy, pneumonectomy and bronchial sleeve resection. The role of other treatment methods has yet to be defined; however, radiochemotherapy is recommended in cases of incomplete resection and malignancy (1). A definitive diagnosis of PPL is provided following pathological examination of a tumor sample. Early detection and complete surgical resection of PPL have been demonstrated to significantly contribute to an

Correspondence to: Professor Xiaoying Huang, Pulmonary Division, The First Affiliated Hospital of Wenzhou Medical University, South Baixiang Street, Wenzhou, Zhejiang 325000, P.R. China

E-mail: zjwzhxy@126.com

Key words: primary pulmonary leiomyosarcoma, chemotherapy increased survival time of patients with the disease (3). Written informed consent was obtained from the patient.

\section{Case report}

A 48-year-old man was admitted to the First Affiliated Hospital of Wenzhou Medical University (Wenzhou, Zhejiang, China) in April 2014 with a cough and the expectoration of white and yellow sputum, which had been ongoing for 2 months. The patient had been a smoker for $>20$ years, but had no family history of lung cancer. The patient also felt short of breath when ascending stairs. The patient did not present with a fever, anorexia, hemoptysis, chest pain or weight loss. The vital signs of the patient were stable. Lung auscultation revealed decreased breath sounds on the right lung and fine crackling was heard at the base of the right lung. Other physical examinations were unremarkable. Blood tests revealed that the patient's white blood count was $9.9 \times 10^{9}$ cells $/ 1$ (normal range, $4.0-10.0 \times 10^{9}$ cells $/ 1$ ), the C-reactive protein level was $48.8 \mathrm{mg} / \mathrm{l}$ (normal range, $0-10 \mathrm{mg} / \mathrm{l}$ ) and the erythrocyte sedimentation rate was $45 \mathrm{~mm} / \mathrm{h}$ (normal range in men, $0-15 \mathrm{~mm} / \mathrm{h}$ ). Other laboratory examinations were all negative, including those for blood chemistry, blood tumor markers, and liver and renal function. Sputum from the patient was negative for acid-fast bacilli and sputum culture.

Enhanced computed tomography (CT) scans of the patient's chest revealed the presence of a $6.6 \times 5.2-\mathrm{cm}$ irregular heterogeneous mass in the hilar region of the right lung, with mediastinal lymphadenopathy and intrapulmonary metastasis (Fig. 1). The CT scan demonstrated that there was a high-density shadow, $8 \mathrm{~mm}$ in diameter (Fig. 2A), on the upper lobe of the right lung and a high-density shadow, $4 \mathrm{~mm}$ in diameter, on the upper lobe of the left lung (Fig. 2B). A whole-body bone scan using positron emission tomography-CT demonstrated that the lung cancer had metastasized to the bone, with abnormal radioactive concentrations observed in the shoulder blades. There were no metastatic lesions observed on abdominal CT and magnetic resonance imaging (MRI) of the brain.

Bronchoscopy revealed visible neoplasms in the lumen of the respiratory tract, which caused the lumen to become decreased in diameter (Fig. 3A). A resection of the right upper lobe tumor of the right total bronchus using a flexible bronchoscope was successfully performed in the patient (Fig. 3B). Subsequently, the patient was administered with systemic chemotherapy targeting the intrapulmonary and bone metastases 15 days subsequent to 


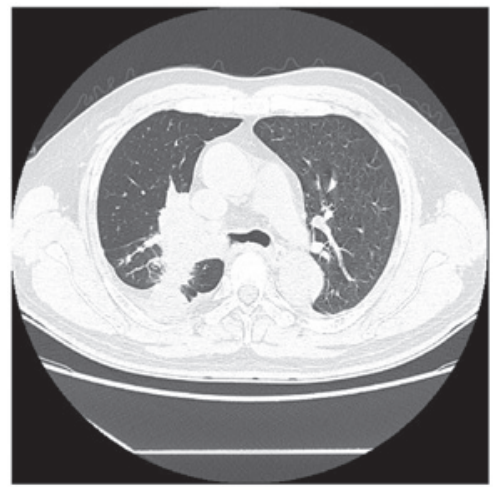

Figure 1. Enhanced computed tomography scans of the chest revealing the presence of a large irregular mass extending into the right pulmonary bronchi, with circumferential narrowing of the upper lobe and anterior segmental bronchus.

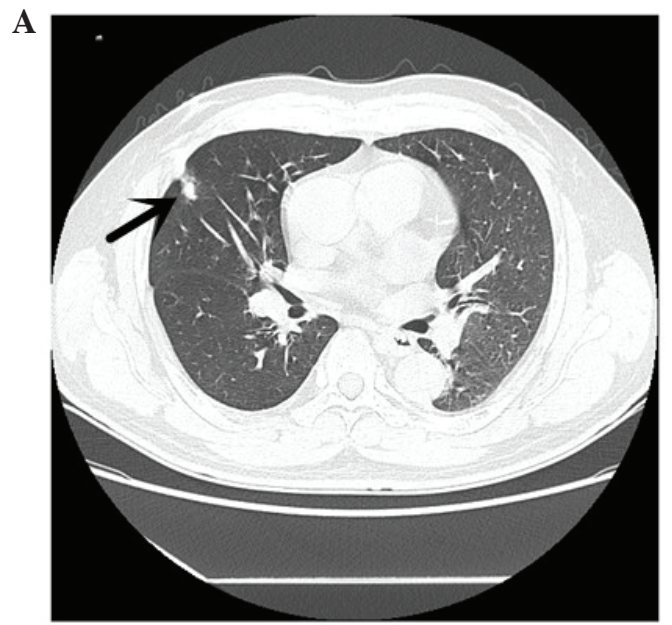

B

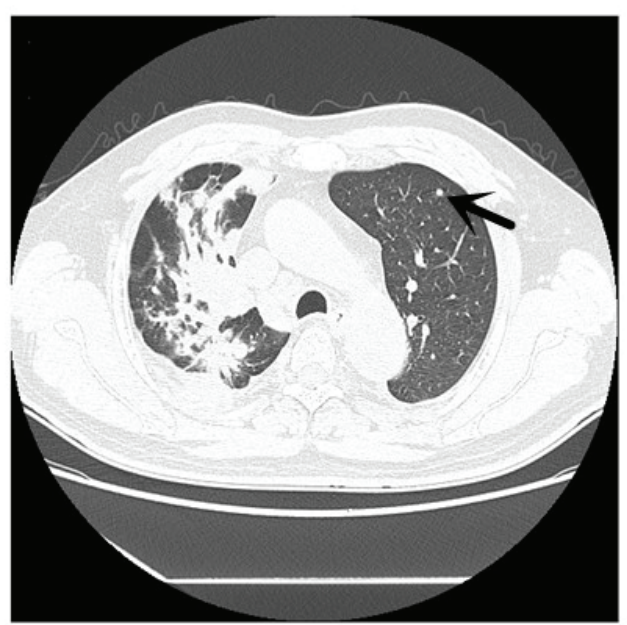

Figure 2. (A) Bronchial 3-dimensional reconstruction. (B) Computed tomography coronal plane of the chest.

resection, as follows: $110 \mathrm{mg}$ epirubicin intravenously for 1 day; and $2.8 \mathrm{~g}$ ifosfamide intravenously for 3 days. The chemotherapy cycle was 21 days. However, the patient succumbed to the disease 2 weeks after the second cycle of chemotherapy.

A biopsy of the resected tumor demonstrated that the tumor was composed of spindle cells, with marked nuclear pleomorphism and numerous mitotic figures (hematoxylin and

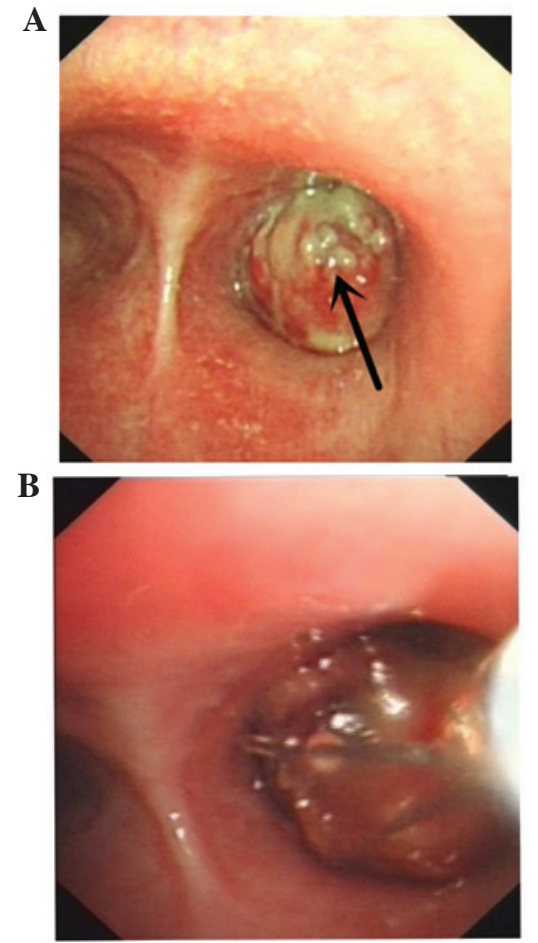

Figure 3. Bronchoscopy images (A) prior to surgery, revealing visible neoplasms in the respiratory tract lumen (black arrow), which caused the lumen to become decreased in diameter, and (B) following successful resection with a flexible bronchoscope.

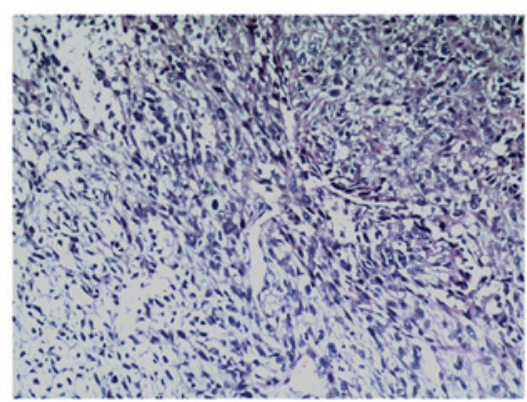

Figure 4. Hematoxylin and eosin staining of the resected primary pulmonary leiomyosarcoma, composed of spindle cells arranged in interweaving bundles. Magnification, x200.

eosin staining; Fig. 4). There was no epithelial differentiation observed in the tumor. Immunohistochemical staining demonstrated that the tumor expressed vimentin, smooth muscle actin, cluster of differentiation 34 (CD34) and actin, and did not express high and low molecular weight cytokeratins, epithelial membrane antigen, desmin and protein S-100. The cell proliferation index of the tumor using antigen Ki-67 was $30 \%$. There was no epithelial differentiation observed in the tumor and the overall morphological features favored a high-grade sarcoma with evidence of smooth muscle differentiation, indicating a leiomyosarcoma. Thus, the final diagnosis was PPL.

\section{Discussion}

PPL is a mesenchymal tumor that has been observed to originate from the smooth muscle cells of the bronchial or 
blood vessel wall. PPL accounts for $<0.5 \%$ of all malignant pulmonary tumors (1), and is classified by type: Intraluminal, intrapulmonary and pulmonary vascular (4). The intrapulmonary type of PPL is the most common, and the pulmonary vascular type originates from the vascular wall and occurs in the pulmonary artery, where it may cause stenosis and obstruction (5). The PPL possessed by the patient in the present study was the intraluminal type.

The majority of patients with PPL present with symptoms similar to those observed in other primary pulmonary tumors, including the presence of a cough, chest pain, dyspnea, hemoptysis and asthenia (1). In order to differentiate PPL from bronchogenic carcinoma, an excisional biopsy is required. Vimentin is present in the majority of mesenchymal cells and is a good mesenchymal tumor marker, which may additionally be used in the identification of sarcoma and carcinoma. Cytokeratin is an epithelial marker. Therefore, vimentin and cytokeratin may be used together to distinguish between the majority of epithelial and mesenchymal tumors $(6,7)$. In the present case, immunohistochemical staining demonstrated that the tumor expressed vimentin, smooth muscle actin, cluster of differentiation 34 and actin, and did not express high and low molecular weight cytokeratins. A diagnosis of PPL should only be considered if signs of an occult tumor are not noted in any other bodily location. In women, it is particularly important to observe if there is a tumor present in the uterus (8). Following a tissue biopsy that confirms PPL, pre-operative staging of the tumor may be considered. Generally, this consists of CT of the lungs and MRI of the primary lesion to determine if the tumor has metastasized, which is uncommon in leiomyosarcomas and is typically observed in the advanced stages of the disease (8).

Leiomyosarcomas are characterized grossly by a firm grey or white surface. Microscopically, malignant spindle cells with cigar-shaped nuclei, arranged in interweaving fascicles, are noted (9). Common features that PPL cells possess are mitotic figures, multinucleation, nuclear atypia, prominent vascularity, scanty cytoplasm and zonal necrosis. Calcification, cavitation, pleural effusions and pneumothorax are not often observed (9). PPL expresses actin, smooth muscle actin, desmin and vimentin, which may be detected using immunohistochemistry and antibodies to these proteins. Generally, leiomyosarcomas do not express carcinoembryonic antigen, cytokeratin, leukocyte common antigen, neuroendocrine filament and S100 protein (10). In the present case, analysis of the resected tumor biopsy demonstrated that the tumor was composed of spindle cells, with marked nuclear pleomorphism and numerous mitotic figures. There was no epithelial differentiation observed in the tumor. Immunohistochemical staining identified expression of vimentin, smooth muscle actin, CD34 and actin in the tumor, however, high and low molecular weight cytokeratins, epithelial membrane antigen, desmin and S100 protein were not expressed. Therefore, the pathological and immunohistochemical results observed in the present study are consistent with these aforementioned characteristics.

Treatment regimens for patients with PPL aim to achieve local and systemic control of the tumor, while preserving function and quality of life. If pre-operative staging demonstrates that there is no evidence of metastases, surgery is recommended, such as a lobectomy and pneumonectomy, which require resection of the chest wall, diaphragm or vascular structures (9). If an early complete resection is performed, the 5-year survival rate of patients is $\sim 50 \%$, and there have been reports of patient survival 20 years post-resection (10). Adjuvant radio- or chemotherapy treatment is recommended in cases of incomplete resection, unresectable tumors and patients with increased histological malignancy; however, it has been demonstrated that radio- and chemotherapy do not extend the survival time of the patient (11). Prognostic indicators of PPL consist of tumor size, extent of bronchial invasion and degree of malignancy (9). In the present study, malignant spindle cells were positive for S100 antigen, smooth muscle actin and vimentin, and were negative for anti-cytokeratin 5.2, caldesmon, cytokeratin $5 / 6$, cytokeratin 7 , cytokeratin 20 , CD34, c-Kit, desmin, epithelial membrane antigen, myogenin, pancytokeratin and thyroid transcription factor-1. Therefore, the results of the present study are consistent with the existing literature (10).

In conclusion, PPL is a rare tumor that grows rapidly. It may be challenging to differentiate PPL from other pulmonary tumors due to the lack of specific manifestations. A pre-operative diagnosis of PPL is considered following the results of a sputum smear, lung biopsy or bronchoscopic examination; however, diagnosing PPL from these methods may also be challenging. In order to differentiate a primary pulmonary leiomyosarcoma from bronchogenic carcinoma, an excisional biopsy is required. Therefore, the most reliable methods for detection and diagnosis of PPL are chest radiography and post-operative pathological examination. The present case emphasizes the important role of pathological and immunohistochemical results in differentiating between PPL and bronchogenic carcinoma. The goal of treatment is to obtain local and systemic control of the sarcoma, while preserving functioning and quality of life. If preoperative evaluation reveals no evidence of metastases, then treatment is surgical. However, if preoperative evaluation reveals evidence of metastasis, the radiation therapy, chemotherapy or a combination of the two is required. An increased awareness of PPL leading to an early diagnosis and the performance of a complete surgical resection with adjuvant radio- and chemotherapy in selected patients may improve the prognosis of patients with PPL.

\section{Acknowledgements}

The authors would like to thank the Records System and Medical Imaging Division of the First Affiliated Hospital of Wenzhou Medical University for collecting the data and figures used by the present study.

\section{References}

1. Rozada R, Vila A and Sosa L: Primary leiomyosarcoma of the lung. Arch Bronconeumol 46: 338-339, 2010 (In Spanish).

2. Janssen JP, Mulder JJ, Wagenaar SS, Elbers HR and van den Bosch JM. Primary sarcoma of the lung: A clinical study with long-term follow-up. Ann Thorac Surg 58:1151-1155, 1994.

3. Shen W, Chen J, Wei S, Wang X, Li X and Zhou Q: Primary pulmonary leiomyosarcoma. J Chin Med Assoc 77: 49-51, 2014.

4. Yu HQ, Ren H, Miao Q, Wang Z, Zhang Z and Xu L: Pulmonary leiomyosarcoma. Chin Med Sci J 12: 129-131, 1997.

5. Pain JA and Sayer RE: Primary leiomyosarcoma of the pulmonary artery. Eur J Respir Dis 65: 139-143, 1984. 
6. Hartel PH, Fanburg-Smith JC, Frazier AA, Galvin JR, Lichy JH, Shilo K and Franks TJ: Primary pulmonary and mediastinal synovial sarcoma: A clinicopathologic study of 60 cases and comparison with five prior series. Mod Pathol 20: 760-769, 2007. 7. Nath D, Arava S, Joshi P, Madan K and Mathur S. Primary pulmonary leiomyosarcoma of lung: An unusual entity with brief review. Indian J Pathol Microbiol 58: 338-340, 2015.

8. Ramanathan T: Primary leiomyosarcoma of the lung. Thorax 29 : 482-489, 1974.
9. Cordes BG, Collins BT, McDonald JW, Khosla A and Salimi Z: Fine needle aspiration biopsy of primary leiomyosarcoma arising from a pulmonary vein. Acta Cytol 43: 523-526, 1999.

10. Arnold LM III, Burman SD and O-Yurvati AH: Diagnosis and management of primary pulmonary leiomyosarcoma. J Am Osteopath Assoc 110: 244-246, 2010.

11. Shaw RR, Paulson DL, Kee JL and Lovett VF: Primary pulmonary leiomyosarcomas. J Thorac Cardiovasc Surg 41: 430-436, 1961. 